\title{
L'ablation/ionisation laser appliquée à la préparation et à l'étude des fullerènes
}

\author{
E. Millon, B. Kubler et J.-F. Muller
}

Laboratoire de Spectrométrie de Masse et de Chimie Laser, Université de Metz, IPC, Technopôle, Metz 2000, 1 boulevard Arago, 57078 Metz cedex 03, France

\begin{abstract}
Résumé : Les fullerènes (molécules carbonées sphéroïdales de formule générale $C_{n} ; n>20$ ) sont classiquement obtenus dans les extraits de suies en mélange avec notamment des hydrocarbures aromatiques polycycliques (HAP) ce qui rend leur détection plus difficile. Nous avons développé une méthodologie d'analyse semi-quantitative par microsonde laser couplée à la spectrométrie de masse afin de caractériser les fullerènes "classiques" $\left(\mathrm{C}_{60}\right.$ et $\left.\mathrm{C}_{70}\right)$. Cette technique permet en outre de contribuer à l'analyse et à l'étude des fullerènes supérieurs $(n>100)$ qui peuvent dans certains cas résulter de la coalescence de fullerènes plus petits.
\end{abstract}

\section{INTRODUCTION}

Les fullerènes sont des molécules carbonées de structure géodésique de formule générale $C_{n}(n>20)$. Quelle que soit la valeur de $n$, le fullerène est constitué de l'association de 12 pentagones isolés et d'un nombre défini d'hexagones : 20 pour $\mathrm{C}_{60}$ et 25 pour $\mathrm{C}_{70}$. Les distributions de fullerènes observées lors de l'analyse de suies révèlent systématiquement l'émergence de ces deux fullerènes $\mathrm{C}_{60}$ et $\mathrm{C}_{70}$ qui sont, à l'heure actuelle, des molécules produites en quantité macroscopique.

Historiquement, la première méthode qui a permis la mise en évidence des fullerènes est l'irradiation d'une cible carbonée (le graphite) par un faisceau laser suivie de la détection des espèces ablatées par spectrométrie de masse [1]. Puis, sont apparues au début des années 90 deux méthodes qui sont désormais largement utilisées pour produire les fullerènes : il s'agit d'une part, de la préparation de suies à partir d'une décharge électrique entre deux électrodes de graphite en atmosphère confinée [2] et d'autre part, de la combustion d'hydrocarbures en défaut d'oxygène (flammes fuligineuses) [3]. Dans les trois cas, les fullerènes sont obtenus en présence d'autres structures carbonées (suies et pour la méthode par flammes, des hydrocarbures aromatiques polycycliques ) avec des rendements compris entre 5 et $20 \%$ en poids variant selon la méthode employée et les conditions expérimentales utilisées. L'obtention de fullerènes purs passe alors par une étape d'extraction par des solvants organiques (le plus classiquement utilisé demeure le toluène) suivie d'une ou plusieurs étapes de séparation par des méthodes chromatographiques qui engendrent un accroissement du prix de revient de ces molécules. Si la préparation des fullerènes $\mathrm{C}_{60}$ et $C_{70}$ de bonne pureté $(>99,9 \%$ ) ne pose plus de problème particulier, il n'en est pas de même pour les fullerènes exotiques tels que les fullerènes supérieurs $(n>70)$ ou dopés par des métaux. A titre d'exemple un gramme de $\mathrm{C}_{60}$ est vendu environ $500 \mathrm{~F}$ contre $100000 \mathrm{~F}$ pour un gramme de $\mathrm{C}_{84}$.

Si la voie "laser" a progressivement laissé la place aux deux autres techniques plus adaptées à la préparation "massive" des fullerènes (arc électrique et combustion d'hydrocarbures), elle reste un procédé intéressant et avantageux pour les raisons 
suivantes:

- la synthèse de fullerènes par ablation laser à différentes longueurs d'onde d'irradiation (UV ou visible) de cibles carbonées ou mixtes (carbone-métal) peut conduire en particulier à la préparation de fullerènes endohédriques (dopage du fullerène par un métal à l'intérieur de la cage géodésique);

- la possibilité d'étudier in-situ les agrégats de carbone par microsondes laser couplées à la spectrométrie de masse (à temps de vol [4] et à résonance cyclotronique des ions [5]), ce qui permet d'accéder aux mécanismes de formation des fullerènes en fonction des conditions d'irradiation laser (irradiance et longueur d'onde).

Ainsi, la caractérisation et la détection des fullerènes sont nécessaires pour évaluer la faisabilité d'une méthode de production et pour étudier leur réactivité.

\section{MISE EN EVIDENCE DES FULLERENES PAR IRRADIATION LASER COUPLEE A LA SPECTROMETRIE DE MASSE}

La première étude d'agrégats carbonés de hautes masses issus de la vaporisation laser de graphite a été réalisée en 1984 par Rohlfing et al. [6]. Les spectres de masse que les auteurs ont obtenus directement après irradiation de la cible (méthode dite "in-situ") se singularisent par la présence de deux distributions d'agrégats. La première présente des agrégats séparés d'une masse de 12 (n est pair et impair) et elle est centrée vers 20 atomes de carbone. La seconde, plus large, présente deux maxima à 60 et 70 atomes de carbone correspondant au centre d'une distribution d'agrégats carbonés tels que n est pair et varie de 40 à 120 . Ce type de distribution sera retrouvée de nombreuses fois ultérieurement mais malheureusement pour ces auteurs, la découverte des fullerènes ne leur sera pas attribuée puisqu'ils n'ont pu proposer de structure aux agrégats observés.

En 1985, Kroto et Smalley [1] irradient un disque tournant de graphite par un faisceau laser Nd YAG à $532 \mathrm{~nm}$ avec une énergie de l'ordre de $30 \mathrm{~mJ}$ par impulsion. Le plasma produit est balayé par un jet d'helium supersonique tangentiel à la surface du disque engendrant ainsi un taux de collisions important. Les agrégats formés, majoritairement neutres, sont dirigés vers une microsonde laser où ils sont photo-ionisés par un laser eximère à $193 \mathrm{~nm}\left(1 \mathrm{~mJ} / \mathrm{cm}^{2}\right)$. Les ions générés sont détectés par spectrométrie de masse à temps de vol (TOF/MS). C'est à l'issue de ces essais que Kroto et Smalley propose un nom et une structure à $\mathrm{C}_{60}$.

De nombreux travaux basés sur la détection "in-situ" des fullerènes à partir de l'ablation laser de matériaux carbonés, ont succédé à cette étude. En 1988, Cox et al. optimisent la procédure précédemment décrite en ajoutant un réacteur de collisions [7]. Greenwood et al. réalisent en 1990 une étude importante concernant la formation des agrégats carbonés à partir de l'irradiation par un laser à $1064 \mathrm{~nm}$ de matériaux carbonés (graphite, charbon, lignite et hydrocarbures aromatiques polycycliques). La distribution des agrégats observés par spectrométrie de masse à résonance cyclotronique des ions (FT/ICR : Fourier Transform Ion Cyclotron Resonance) montre que la formation de $\mathrm{C}_{60}$ est fonction du rang du charbon [8]. En 1991, Dance et al. [9] appliquent cette méthode à l'étude des mésophases (structures charbonneuses préorientées) et à l'effet de l'atmosphère de confinement.

\section{SYNTHESE DE FULLERENES PAR ABLATION LASER DE MATERIAUX CARBONES}

La première méthode de préparation de fullerènes à l'échelle macroscopique par laser est publiée par Meijer et Bethune en 1990 [10]. L'irradiation laser à $532 \mathrm{~nm}$ d'une cible de graphite dans une enceinte en atmosphère confinée d'argon (500 Torrs) conduit à un dépôt de suies sur un substrat. Le principe de l'ablation laser est le suivant. Le dépôt est 
obtenu par ablation laser suivi du transfert des éléments constitutifs de la cible vers le substrat lors de l'expansion du "plasma". Ce dernier peut être défini comme un panache de matière constitué majoritairement d'espèces neutres (molécules, atomes) à l'état fondamental ou excité et en proportion moindre ( 1 pour 1000 à 1 pour 10000 ) d'ions et d'électrons. Le dépôt est ensuite récupéré puis analysé en dehors de l'enceinte de préparation (on parle alors d'analyse "ex-situ") par spectrométrie de masse.

Au cours de leurs travaux, Meijer et Bethune soulignent l'ambiguité qui subsiste quant à l'endroit où sont effectivement produits les fullerènes : les fullerènes sont-ils formés lors de l'ablation laser du graphite dans l'enceinte réactionnelle ou lors de l'analyse du dépôt par spectrométrie de masse? Pour répondre à cette question, ils effectuent des ablations sur différentes cibles constituées de ${ }^{12} \mathrm{C}$ pur (cas a), de ${ }^{13} \mathrm{C}$ pur (cas b), d'un mélange équimolaire de ${ }^{12} \mathrm{C}$ et ${ }^{13} \mathrm{C}$ (cas c) et enfin de couches successives de ${ }^{12} \mathrm{C}$ et ${ }^{13} \mathrm{C}$ (cas $\mathrm{d}$ ). Si la synthèse des fullerènes était effective au sein de la microsonde laser, des spectres de masse équivalents auraient dû être observés lors de l'analyse des dépôts obtenus pour les essais c et d. Ceci n'étant pas le cas, les auteurs prouvent bien la formation de fullerènes par ablation laser de graphite sous atmosphère confinée.

Nous avons appliqué ce mode de préparation à l'étude de différents matériaux carbonés cible. Du graphite, de la mésophase, un brai pétrolier et un charbon bitumineux ont été successivement irradiés par un faisceau laser à $532 \mathrm{~nm}$ dans une enceinte d'ablation confinée sous 500 torrs d'argon [11]. Les dépôts obtenus ont été analysés par microsonde laser couplée à la spectrométrie de masse à temps de vol LAMMA 500 [4]. A titre d'exemples, deux spectres de masse réalisés en mode d'ionisation positif sont donnés figure 1. Ils correspondent aux dépôts obtenus par ablation laser du graphite (matériau carboné de rang le plus élevé) et du charbon bitumineux (rang le plus faible).
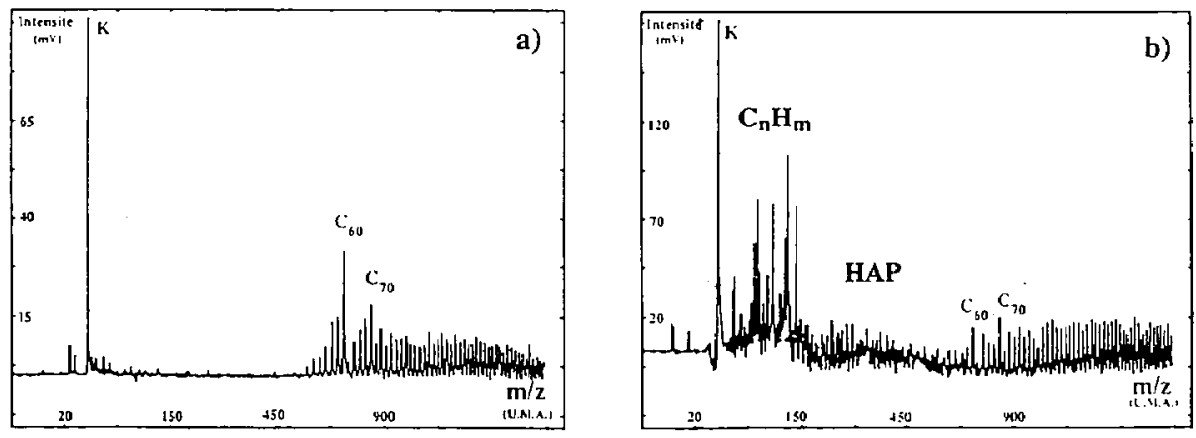

Figure 1 : spectres LAMMA à $266 \mathrm{~nm}$ (ions positifs) d'un dépôt obtenu par ablation laser à $532 \mathrm{~nm}$ de graphite (a), de charbon bitumineux (b)

Les distributions des agrégats carbonés diffèrent notablement d'une cible à l'autre et on pourrait penser, au vu de ces résultats, que la nature chimique du matériau cible influe sur la proportion relative de $C_{60}$ et $C_{70}$ : en effet le rapport $C_{70} / C_{60}$ est voisin de 0,6 pour le graphite et de 1,1 pour le charbon. En fait, il n'en est rien car comme nous le verrons ultérieurement, la distribution des agrégats ionisés en mode positif est perturbée par la présence d'hydrocarbures aromatiques polycycliques (HAP) dans le matériau cible. Ces derniers sont d'ailleurs détectés sur le spectre de masse correspondant à l'ablation du charbon (figure $\mathrm{lb}$ ). Le problème de la détection et à 
fortiori du dosage des fullerènes dans un matériau complexe (suie en présence d'HAP par exemple) par spectrométrie de masse se pose donc.

\section{ANALYSE DES FULLERENES PAR MICROSONDE LASER}

L'analyse LAMMA d'un matériau carboné quelconque conduit à des spectres de masse en mode positif ou négatif sur lesquels apparaissent schématiquement trois types d'ions dans des proportions relatives variables en fonction des conditions expérimentales d'analyse (irradiance et longueur d'onde du laser en particulier) :

- des agrégats de recombinaisons de type $\mathrm{C}_{n} \mathrm{H}_{m}{ }^{+}$ou $\mathrm{C}_{n}{ }^{-}(\mathrm{n}<15$ et $\mathrm{m}<3)$ de masses inférieures à 150 environ ;

- des pics caractéristiques de molécules ionisées mais non fragmentées (pics moléculaires) dus notamment à la présence d'espèces organiques telles les HAP ;

- et dans certaines conditions, des pics de hautes masses $(\mathrm{m} / \mathrm{z}>400)$ de type $\mathrm{C}_{\mathrm{n}}{ }^{+/-}$dont la distribution relative peut correspondre à celle des fullerènes à structure fermée $\left(C_{n}\right)$. Une illustration de ces différentes espèces est d'ailleurs montrée figure $1 b$.

Lors de l'analyse d'un matériau carboné, on distingue en général deux régimes d'interaction fonction de la densité de puissance du laser envoyée sur la cible. A faible irradiance $\left(<10^{7} \mathrm{~W} / \mathrm{cm}^{2}\right)$, les phénomènes de désorption sont prépondérants avec une décomposition minimisée des espèces carbonées présentes dans le microplasma. A irradiance plus élevée $\left(10^{8} \mathrm{~W} / \mathrm{cm}^{2}\right)$, l'énergie incidente est suffisante pour casser les molécules de hautes masses en fragments élémentaires carbonés hydrogénés susceptibles de réagir pour former des agrégats de recombinaison. Le plasma est alors le siège de réactions ions-molécules conduisant à la détection d'ions de hautes masses que l'on pourrait attribuer à des fullerènes ou à des molécules sphéroïdales. Il est donc important de distinguer et si possible de quantifier les ions $\mathrm{C}_{n}{ }^{+}$formés "in-situ" lors de l'analyse d'un matériau carboné quelconque de ceux susceptibles d'être issus de fullerènes présents initialement dans le composé analysé. Généralement, le dosage des fullerènes est effectué par une observation des distributions obtenues en ions positifs qui, dans des conditions d'analyse précises, se retrouve aussi en ions négatifs (figure 2).
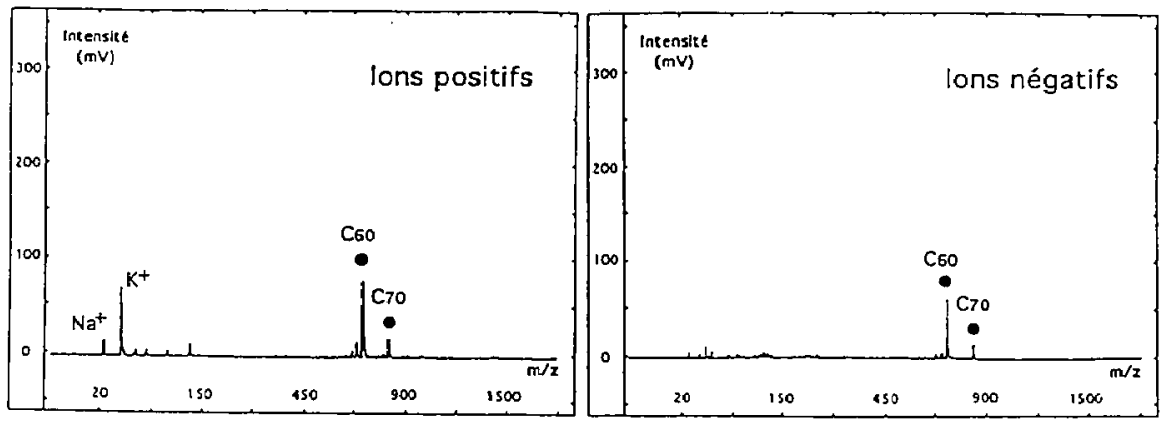

Figure 2 : spectres LAMMA (266 nm, mode désorption) d'un mélange de $\mathrm{C}_{60}{ }^{\prime} \mathrm{C}_{70}\left(85 \% \mathrm{C}_{60}-15 \% \mathrm{C}_{70}\right)$

\subsection{Comportement des HAP lors d'analyses par microsonde laser}

La figure 3 a présente les spectres LAMMA obtenus en ions positifs pour l'analyse de composés aromatiques polycycliques (coronène $\mathrm{C}_{24} \mathrm{H}_{12}$, anthracène $\mathrm{C}_{14} \mathrm{H}_{10}$ ) dans des 
conditions de désorption laser (Irradiance $=10^{6} \mathrm{~W} / \mathrm{cm}^{2}$ ). Sur ces spectres, les ions $\mathrm{C}_{\mathrm{n}}{ }^{+}$ ne sont pas détectés et les ions moléculaires $\mathrm{M}^{+{ }^{\circ}}$ apparaissent nettement pour les composés aromatiques. A irradiance plus élevée, la présence des pics moléculaires des HAP et des agrégats de basses masses $\mathrm{C}_{n} \mathrm{H}_{\mathrm{m}}{ }^{+}$est remarquée (figure $3 b$ ).
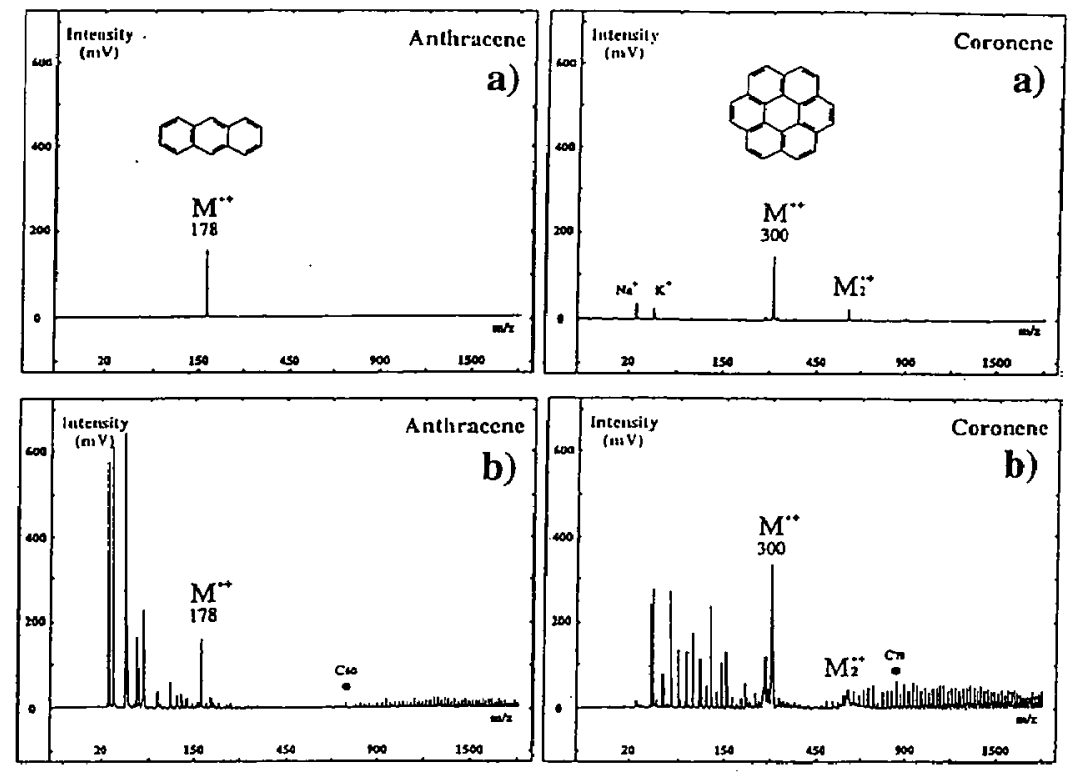

Figure 3: spectres LAMMA en ions positifs à $266 \mathrm{~nm}$ de composés aromatiques : en mode désorption (a) et à forte irradiance $\left(10^{8} \mathrm{~W} / \mathrm{cm}^{2}\right)$ (b)

Ceci peut s'expliquer par le phénomène de "thermal spike" [12] lors de l'ablation laser : l'énergie du faisceau laser à la périphérie du point d'impact étant plus faible, des phénomènes de désorption peuvent avoir lieu. Par contre, au centre de l'impact, il y a formation d'agrégats $\mathrm{C}_{n} \mathrm{H}_{\mathrm{m}}{ }^{+}$. Les deux espèces en présence peuvent alors conduire par réactions ion/molécule aux dimères et trimères correspondant à l'HAP analysé et à la formation d'ions $\mathrm{C}_{\mathrm{n}}{ }^{+}$(figure $3 \mathrm{~b}$ ). Notons que dans ce cas, les distributions des $\mathrm{C}_{\mathrm{n}}{ }^{+}$font apparaitre des agrégats de hautes masses $(>100)$ sans la prédominance nette de $\mathrm{C}_{\mathrm{n}}$ particuliers $(n=60,70,84)$, phénomène rencontré habituellement lors de l'analyse laser de fullerènes purs .

Lorsque les analyses sont effectuées en mode négatif, on constate que le comportement des fullerènes est singulier comparé à celui de leurs homologues aromatiques (HAP). Pour ces derniers, les pics moléculaires $\mathbf{M}^{-}$ne sont jamais remarqués Iors de l'analyse par microsonde LAMMA et les pics $\mathrm{C}_{n}{ }^{-}$ne sont pas détectés lors de l'analyse des composés aromatiques tels que le coronène, l'anthracène et ceci quelles que soient les conditions expérimentales utilisées. L'ensemble de ces résultats a été commenté et expliqué [13]. Contrairement à l'analyse en ions positifs, la détection du pic $\mathrm{C}_{n}{ }^{-}$est indubitablement attribuable à la présence initiale de fullerènes $\left(\mathrm{C}_{60}, \mathrm{C}_{70}\right.$ par exemple) dans le matériau analysé. 


\subsection{Analyse semi-quantitative des fullerènes}

Notre objectif a été d'explorer la possibilité de doser quantitativement et in-situ les fullerènes présents dans des mélanges bruts de synthèse (suies et dépôts). Dans un premier temps, nous avons tenté de doser les fullerènes en utilisant la méthode de l'ajout d'un étalon interne : le coronène. Nous avons alors constaté que la quantification des fullerènes était rendue délicate du fait que la présence du HAP (le coronène) perturbait leur distribution relative par des réactions d'échange au sein du microplasma du type : $\mathrm{HAP}^{+}+\mathrm{C}_{n}---->\mathrm{HAP}+\mathrm{C}_{n}{ }^{+}$

Par contre, pour des mélanges de fullerènes purs, l'analyse LAMMA est très performante puisqu'elle conduit à la détermination des proportions relatives des fullerènes (rapport $C_{70} / C_{60}$ par exemple) avec une précision comparable aux résultats fournis par les techniques chromatographiques et spectroscopiques comme le montre la figure 4.

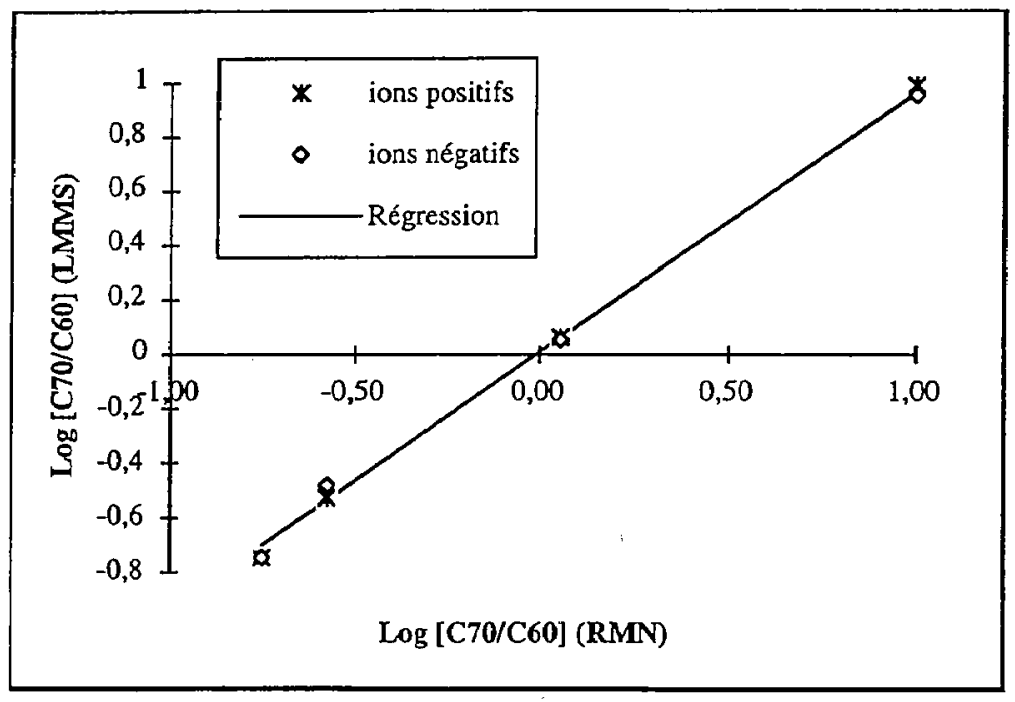

Figure 4 : évolution des rapports des intensités des pics $\mathrm{C}_{60}$ et $\mathrm{C}_{70}$ (ions positifs el négatifs)

En résumé, la présence de fullerènes dans un échantillon se remarquera donc par la détection simultanée des ions $\mathrm{C}_{\mathrm{n}}{ }^{+}$et des ions $\mathrm{C}_{\mathrm{n}}{ }^{-}$. La seule présence des ions $\mathrm{C}_{\mathrm{n}}{ }^{+}$ (sans ions $\mathrm{C}_{\mathrm{n}}{ }^{-}$remarqués sur les spectres correspondants en mode négatif) peut être due à des réactions de formation de structures sphéroïdales ouverte à partir de HAP susceptibles d'être présents également dans l'échantillon. Il en ressort que la présence d'HAP souvent remarquée en mélange avec les fullerènes dans les suies de flammes, perturbe à la fois les intensités des pics $\mathrm{C}_{\mathrm{n}}{ }^{+}$et leur distribution relative, mais cette technique reste parfaitement adaptée à l'analyse qualitative des fullerènes dans ces conditions (détection des agrégats ionisés négatifs). En revanche, le dosage semiquantitatif de fullerènes purs par microsonde LAMMA est parfaitement adapté. Soulignons de plus, que la détection de fullerènes minoritaires ( $\mathrm{C}_{84}$ et fullerènes géants) difficilement mis en évidence par d'autres méthodes est possible dans ce cas, ce qui rend l'analyse par microsonde LAMMA particulièrement attractive. 


\section{APPROCHE MECANISTIQUE DE LA FORMATION DES FULLERENES SUPERIEURS}

La spectrométrie de masse couplée à l'ionisation laser peut se révéler également intéressante pour étudier les agrégats carbonés de hautes masses $(n>100)$ susceptibles d'être détectés lors de l'analyse de suies. Cette méthode permet en outre d'appréhender les processus de fragmentation et de recombinaison des espèces au sein du microplasma.

Ainsi, le comportement de fullerènes purs $\left(\mathrm{C}_{60}, \mathrm{C}_{70}, \mathrm{C}_{76}, \mathrm{C}_{84}\right)$ sous l'influence d'irradiations laser UV (223 et $266 \mathrm{~nm}$ ) et visible $(566 \mathrm{~nm})$ à différentes densités de puissance conduit aux constatations suivantes [14]. Quel que soit le fullerène étudié, les spectres de masse montrent une distribution d'agrégats carbonés de hautes masses $(\mathbf{n}>100)$ lorsque l'irradiance est supérieure au seuil de désorption des fullerènes $\left(>10^{6} \mathrm{~W} / \mathrm{cm}^{2}\right)$. Ces agrégats sont le fruit de coalescences entre le fullerène initial et ses fragments (issus de la perte ou de l'adjonction de motifs $\mathrm{C}_{2}$ ). La nature des produits coalescés dépend fortement de la longueur d'onde d'irradiation. Pour une irradiation UV, les réactions de fragmentation sont suffisamment importantes pour conduire à des agrégats de type fullerènes "ouverts" $C_{n-2 m}(n=60,70,76,84 ; m<10)$ qui vont coalescer entre eux ou avec le fullerène de départ. Par contre, pour une irradiation visible, des réactions thermiques sont favorisées et conduisent à la formation d'agrégats dopés en groupements $C_{2}\left(C_{n+2 p} ; p<10\right)$. Les distributions obtenues sont alors déplacées vers les hautes masses.

Ceci signifie que les distributions d'agrégats carbonés de hautes masses éventuellement observées sur les spectres de masse peuvent indiquer, soit la présence de fullerènes supérieurs ayant effectivement la structure géodésique, soit la formation d'agrégats ayant coalescés directement au sein du plasma durant l'analyse. Une fois encore, il est mis en évidence que l'analyse de tout matériau susceptible de contenir des fullerènes (classiques ou supérieurs) doit être menée à faible irradiance (désorption laser).

\section{CONCLUSIONS}

La troisième variété allotropique du carbone que constituent les fullerènes peut être préparé par ablation laser de matériaux carbonés. Si cette voie semblait à l'origine prometteuse pour synthétiser spécifiquement un fullerène, elle a été rapidement concurencée par des techniques plus rentable (l'arc électrique et la flamme) pour la préparation des fullerènes classiques $\left(\mathrm{C}_{60}\right.$ et $\left.\mathrm{C}_{70}\right)$. Néanmoins, l'ablation laser demeure intéressante pour des productions particulières tels que les fullerènes dopés endohédriques. D'un point de vue analytique, nous avons vu que l'ionisation laser couplée à la spectrométrie de masse (à temps de vol ou à résonance cyclotronique) s'avère une technique très performante pour la détection qualitative des fullerènes dans une matrice complexe si les conditions expérimentales (irradiance) sont bien maîtrisées. De plus, elle permet d'effectuer des dosages semi-quantitatifs d'un mélange de fullerènes purs et d'appréhender les mécanismes de formation de ces molécules au sein du microplasma laser.

\section{Références}

[1] Kroto H.W., Heath J., O'Brien S.C., Curl R.F., Smalley R.E., Nature 318 (1985) 168

[2] Krätschmer W., Lamb L., Fostiropoulos K., Huffman D.R., Nature 347 (1990) 354

[3] Gerhardt P. , Löfler S., Homan K.H., Chem. Phys. Lett. 137 (1987) 306

[4] Krier G., Muller J.F., La Revue de Métallurgie (1987) 311

[5] Muller J.F., Pelletier M., Krier G., Weil D., Campana J., Microbeam Analysis 
(San Francisco Press 1989) 311

[6] Rohlfing E.A., Cox D.M., Kaldor A., J. Chem. Phys. 81 (1984) 3322

[7] Cox D.M., Reichmann K.C., Kaldor A.,J. Chem. Phys. 88 (1988) 1588

[8] Greenwood P.F., Strachan M.G., El-Nakat H.J., Willet G.D., Wilson M.A., Attalla M.I., Fuel 69 (1990) 257

[9] Dance I.G., Fisher K.J., Willett G.D., Wilson M.A., J. Chem. Phys. 95 (1991) 8425

[10] Meijer G., Bethune D.S., J. Chem. Phys. 93 (1990) 7800

[11] Millon E., Weber J., Théobald J., Muller J.F., C.R. Acad. Sci. Paris 315 (1992) 947

[12] Hercules D.M., Day R.J., Balasanmagan K., Dong T.A., Li C.P., Anal. Chem. 54 (1982) $280 \mathrm{~A}$

[13] Millon E., Weber J., Kubler B., Théobald J., Muller J.F., Analusis 21 (1993) 319

[14] Kubler B., Millon E., Gaumet J.J., Muller J.F., Full. Sci. Tech. (1996) sous presse 